\title{
Pancreatitis aguda en el lupus eritematoso sistémico de inicio en la niñez: A propósito de un caso
} Acute pancreatitis in childhood-onset systemic lupus erythematosus: Case report

\author{
Dr. Yu-feng $L i^{a}$ y Dr. Min-jiang Wei ${ }^{a}$
}

\begin{abstract}
RESUMEN
El lupus eritematoso sistémico (LES) es una enfermedad multisistémica con manifestaciones proteicas. La pancreatitis lúpica es la segunda enfermedad más frecuentemente asociada con el abdomen agudo en relación con el LES. Si bien la pancreatitis aguda es rara, es clínicamente importante porque puede ser potencialmente mortal si no se trata de inmediato. En este artículo, describimos el caso de una niña de 10 años que desarrolló pancreatitis asociada a LES después del tratamiento con corticoesteroides que se complicó posteriormente debido a septicemia fúngica. Los signos y síntomas clínicos mejoraron marcadamente después de la administración de glucocorticoides y ciclofosfamida.

Palabras clave: lupus eritematoso sistémico, pancreatitis aguda, infección micótica, septicemia, niño.
\end{abstract}

http: / / dx.doi.org/10.5546/ aap.2019.e279

Texto completo en inglés:

http: / / dx.doi.org/10.5546/ aap.2019.eng.e279

Cómo citar: Li YF, Wei MJ. Pancreatitis aguda en el lupus eritematoso sistémico de inicio en la niñez: A propósito de un caso. Arch Argent Pediatr 2019;117(3):e279-e283.

\section{INTRODUCCIÓN}

El lupus eritematoso sistémico (LES) es una enfermedad multisistémica con manifestaciones proteicas. El dolor abdominal es un síntoma común en los pacientes con compromiso gastrointestinal relacionado con LES. ${ }^{1}$ La pancreatitis lúpica es la segunda enfermedad más frecuentemente

a. Departamento de Nefrología Pediátrica, Hospital Xinhua afiliado a la Facultad de Medicina de la Universidad de Shanghái Jiao Tong, China.

Correspondencia:

Dr. Yu-feng Li: liyufeng@xinhuamed.com.cn

Financiamiento: Ninguno.

Conflicto de intereses: Ninguno que declarar.

Recibido: 24-7-2018

Aceptado: 10-12-2018 asociada con el abdomen agudo relacionado con LES. $^{2}$ Se observa compromiso gastrointestinal en el $19,4 \%$ de los pacientes pediátricos con LES, mientras que la pancreatitis asociada a LES se produce en el 6,0 \% de los pacientes pediátricos con LES. ${ }^{3} \mathrm{Si}$ bien la pancreatitis aguda es rara, ${ }^{4,5}$ es de relevancia clínica porque puede ser potencialmente mortal si no se trata de inmediato. En este artículo, describimos el caso de una niña pequeña que desarrolló pancreatitis asociada a LES con una complicación posterior debido a septicemia fúngica.

\section{Caso}

Una niña de 10 años presentó, en primer lugar, edema palpebral, llagas gingivales y fiebre continua durante más de siete días. No tenía antecedentes de fotosensibilidad. El hígado y el bazo eran palpables a $4 \mathrm{~cm}$ y a $2 \mathrm{~cm}$, respectivamente, por debajo de los ángulos costofrénicos. No se observaron inflamación ni sensibilidad en las articulaciones. En el examen neurológico, no se notaron anomalías. Durante la hospitalización, los análisis de laboratorio iniciales mostraron lo siguiente: hemoglobina $127 \mathrm{~g} / \mathrm{L}$, recuento de leucocitos $1,85 \times 10^{9} / \mathrm{L}$ (neutrófilos 59,8 \%, linfocitos 33,3\%, monocitos $5 \%$ ), recuento de trombocitos $100 \times 10^{9} / \mathrm{L}$, velocidad de sedimentación globular $20 \mathrm{~mm} /$ hora y proteína C-reactiva (PCR) por debajo de $8 \mathrm{mg} / \mathrm{L}$. Las pruebas funcionales hepáticas arrojaron los siguientes resultados: alanina aminotransferasa $48 \mathrm{U} / \mathrm{L}$ (intervalo normal: 0-75 U/L), aspartato aminotransferasa $82 \mathrm{U} / \mathrm{L}$ (intervalo normal: 8-38 U/L), proteínas totales 47,7 g/L (intervalo normal: 63-82 g/L), albúmina 21,2 g/L (intervalo normal: 35-50 g/L), bilirrubina total $2,4 \mu \mathrm{mol} / \mathrm{L}$, triglicéridos $2,54 \mathrm{mmol} / \mathrm{L}$, fosfatasa alcalina $119 \mathrm{U} / \mathrm{L}$, amilasa $87 \mathrm{U} / \mathrm{L}$ (intervalo normal: 30-110 U/L), lipasa $146 \mathrm{U} / \mathrm{L}$ (intervalo normal: 23-300 U/L) y gammaglutamiltranspeptidasa $14 \mathrm{U} / \mathrm{L}$. Las pruebas funcionales renales indicaron un valor de nitrógeno ureico en sangre de $8,98 \mathrm{mmol} / \mathrm{L}$ (intervalo normal: 2,86-7,14 mmol/L) y de creatinina de $66 \mu \mathrm{mol} / \mathrm{L}$ (intervalo normal: 35-97 $\mu \mathrm{mol} / \mathrm{L}$ ). 
En los análisis de orina, se observó proteinuria, con un recuento de 8-10 eritrocitos/ campo de gran aumento. La excreción de proteínas en la orina de 24 horas fue de 3,06 g/ día. Los resultados de las pruebas inmunológicas fueron: anticuerpo antinuclear 1:640, anticuerpos anti-ADN bicatenario positivos, anticuerpos anticitoplasma de neutrófilos positivos, anticuerpos anti-Sm negativos, anticuerpos anti-SS-A y anti-SS-B negativos. La prueba directa de Coombs fue positiva. Las concentraciones de los componentes del complemento eran extremadamente bajas: CH50 2,2 U/L (intervalo normal: 10-65 U/L), C3 0,18 g/L (intervalo normal: 0,9-1,8 g/L) y C4 0,06 g/L (intervalo normal: 0,1-0,4 g/L). No se observaron anomalías en la radiografía de tórax.

Cinco días después de la hospitalización, la paciente tuvo convulsiones súbitas. Las convulsiones se presentaron con pérdida del conocimiento, trismo, cianosis, como convulsiones tónico-clónicas sin fiebre. Inmediatamente, se le realizaron una tomografía computada (TC) de la cabeza y un análisis del líquido cefalorraquídeo (LCR). Los resultados de estos estudios fueron normales. Las convulsiones fueron causadas por hiponatremia (sodio sérico $120 \mathrm{mmol} / \mathrm{L}$ ) o hipocalciemia (calcio sérico $1,56 \mathrm{mmol} / \mathrm{L}$ ). Se ingresó a la paciente a la unidad de terapia intensiva. Los valores de hemoglobina y leucocitos disminuyeron a $94 \mathrm{~g} / \mathrm{L}$ y $0,36 \times 10^{9} / \mathrm{L}$, respectivamente. En la TC se observaron derrame pleural y ascitis. A la paciente se le diagnosticó LES conforme a los criterios diagnósticos del Colegio Estadounidense de Reumatología. No fue posible realizar una biopsia renal debido a la condición de la paciente. Una vez obtenido el diagnóstico, se le administró metilprednisolona en inyección intravenosa $(20 \mathrm{mg} / \mathrm{kg}$ de peso corporal durante tres días consecutivos) a partir del séptimo día de hospitalización seguida de prednisona por vía oral (2 $\mathrm{mg} / \mathrm{kg}$ al día) e inmunoglobulina en dosis altas $(1 \mathrm{~g} / \mathrm{kg}$ de peso corporal por vía intravenosa durante dos días consecutivos). La paciente presentó pancreatitis aguda con dolor abdominal intenso, derrame pleural y ascitis. En los análisis de sangre se observó aumento de la amilasa sérica (192 U / L), de la lipasa sérica (726 U/L) y de la PCR (33 mg/L). En la ecografía del abdomen se observó que el páncreas estaba marcadamente agrandado, aunque no se notaron cálculos biliares. El conducto colédoco no estaba dilatado. En la TC del abdomen se observaron inflamación de la vesícula biliar con edema pericolecístico, además de agrandamiento difuso y realce homogéneo del páncreas, del bazo y del hígado. En la colangiopancreatografía endoscópica retrógrada (CPER), se mostró estrechamiento difuso del conducto pancreático común con múltiples estenosis.

Se administraron otras tres inyecciones intravenosas de metilprednisolona y se agregó inmunosupresión con ciclosporina $(4,5 \mathrm{mg} /$ kg al día). El día 29 de la hospitalización, en el hemocultivo se observó septicemia fúngica (Candida parapsilosis). Se agregó voriconazol (7 mg/kg al día) para el tratamiento de la septicemia fúngica. Durante la hospitalización, se produjeron varias recidivas de la septicemia por Candida parapsilosis. Se consideró que podría tratarse de una septicemia relacionada con el catéter central de inserción periférica (PICC, por sus siglas en inglés), pero después de retirarlo, se descartó la septicemia fúngica. La niña no respondió al tratamiento con glucocorticoides más ciclosporina. En el análisis de orina se observó proteinuria masiva $(15,14 \mathrm{~g} /$ día $)$. Se cambió el tratamiento inmunosupresor a micofenolato mofetilo por vía oral (34 mg/kg al día). Sin embargo, en los resultados de la orina de 24 horas, el valor siguió estando por encima de $10 \mathrm{~g} /$ día. El día 113 de la hospitalización, se administró ciclofosfamida en inyección intravenosa (8$12 \mathrm{mg} / \mathrm{kg} /$ día durante dos días consecutivos cada dos semanas). Se disminuyó progresivamente la dosis de micofenolato mofetilo después de la administración de ciclofosfamida; al igual que la dosis de corticoesteroides, a 1,5 mg/kg al día. En el examen histológico de la muestra obtenida por biopsia por punción del riñón se observó nefritis lúpica proliferativa difusa de clase III y clase $\mathrm{V}$ conforme a la clasificación de la nefritis lúpica de 2003 de la Sociedad Internacional de Nefrología (International Society of Nephrology, ISN)/Sociedad de Patología Renal (Renal Pathology Society, RPS) (ISN/RPS). ${ }^{6}$ Tras cuatro ciclos de ciclofosfamida en inyección intravenosa, la excreción en la orina de 24 horas de la paciente se redujo a $1732 \mathrm{mg}$, y todos los resultados de los análisis de laboratorio mejoraron. Durante la hospitalización, la paciente no recibió nada por boca y se le administraron líquidos intravenosos y nutrición parenteral total hasta que desapareció el dolor abdominal y se normalizaron las enzimas pancreáticas. A los 177 días de hospitalización, la paciente estaba en condiciones de recibir el alta. Si bien, en la resonancia magnética del cerebro realizada el día 42 después del alta, se observaron signos de 
FIgURA 1. Resultados de laboratorio durante el tratamiento de los episodios de pancreatitis

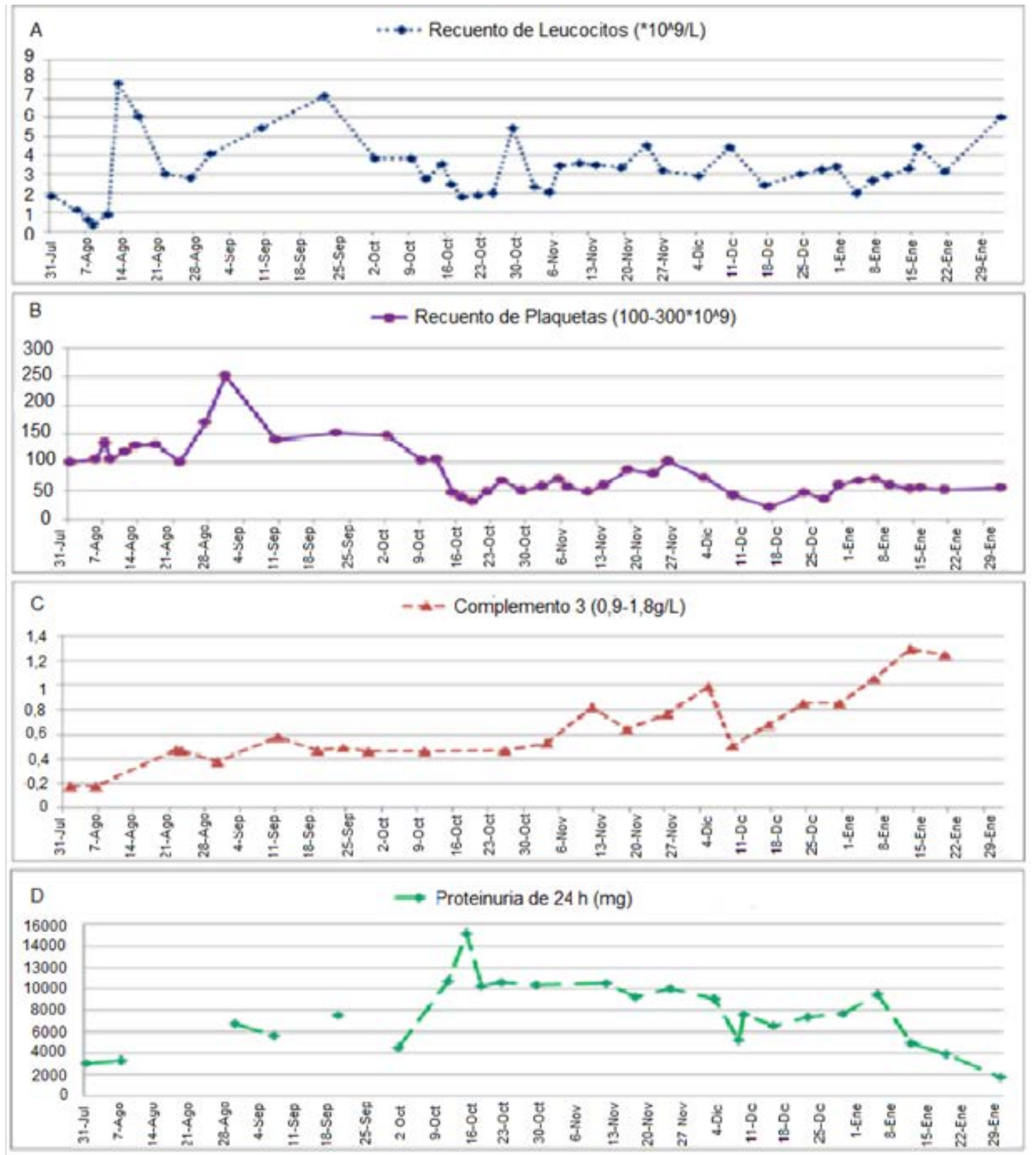

\section{Ciclosporina Micofenolato mofetilo}




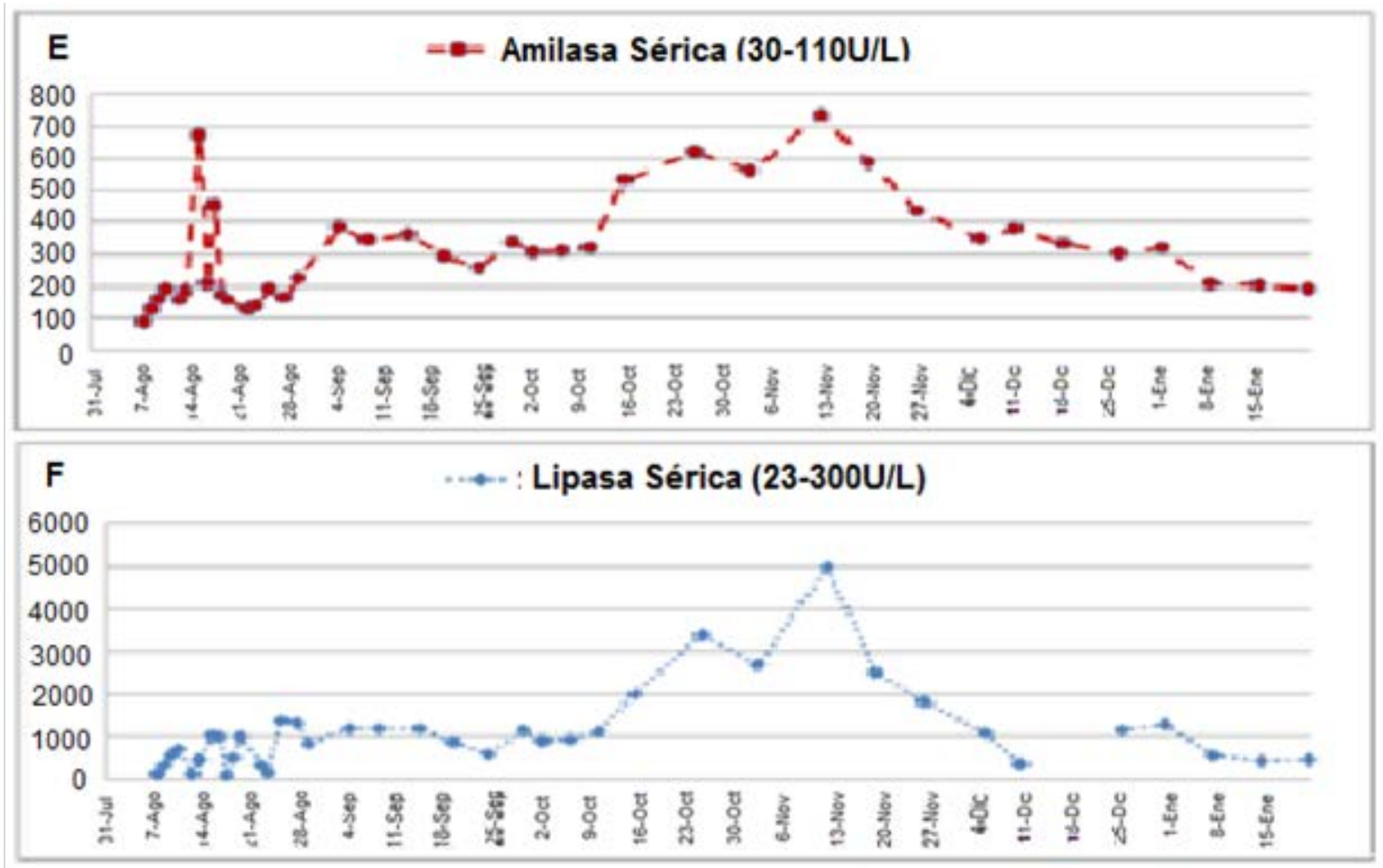

Ciclosporina: 4,5 mg / kg oral por día durante 7 semanas; Micofenolato mofetilo: $34 \mathrm{mg} / \mathrm{kg}$ oral por día durante 10 semanas; Ciclofosfamida: infusión intravenosa ciclofosfamida 8-12 mg / kg / día durante dos días consecutivos cada 2 semanas; Metilprednisolona: metilprednisona oral 1,6 mg / kg por día durante 8 semanas y luego 1,2 mg / kg por día.

atrofia difusa, durante cinco años, la paciente no presentó síntomas. En la actualidad, la paciente no tiene síntomas, recibe corticoesteroides orales en dosis bajas (2,5 mg por vía oral cada dos días) y se le brinda seguimiento en la unidad de atención ambulatoria.

\section{DISCUSIÓN}

El LES es una enfermedad multisistémica que puede afectar casi todos los sistemas y órganos del cuerpo. La prevalencia de la pancreatitis asociada a LES varía del $0,67 \%$ al $4 \%$ en los pacientes con LES. ${ }^{7}$ La incidencia anual de la pancreatitis asociada a LES se estima en $0,4-1,1$ por cada 1000 pacientes con lupus. ${ }^{8}$ La prevalencia de este tipo de pancreatitis es mayor en el caso de LES de inicio durante la niñez $(5,22 \%)$ que en el de inicio durante la adultez. ${ }^{9}$ Aproximadamente el $91 \%$ de los casos de pancreatitis que se diagnostican en pacientes con LES de inicio durante la niñez son de pancreatitis aguda; al contrario, solo el $9 \%$ de esos conducen a pancreatitis aguda recurrente. ${ }^{10}$

La etiología de la pancreatitis asociada a LES sigue siendo poco clara. Se definieron las causas frecuentes de la pancreatitis lúpica como obstrucción mecánica del conducto pancreático (más comúnmente como resultado de coledocolitiasis) y causas tóxicas-metabólicas (secundarias a la ingesta de alcohol, el tratamiento con ciertos fármacos, como corticoesteroides, hiperpotasiemia o hipertrigliceridemia). Era improbable que el tratamiento con corticoesteroides fuera la causa de la pancreatitis observada en la paciente descrita aquí, ya que el tratamiento con dosis altas de corticoesteroides alivió los signos y los síntomas. En este caso, después de excluir otras causas frecuentes de la pancreatitis aguda, a la paciente se le diagnosticó LES con pancreatitis aguda. Los estudios de diagnóstico por imagen, en especial la TC del abdomen, son útiles para el diagnóstico de ciertas gastroenteropatías relacionadas con el LES. Se recomienda la TC para evaluar la gravedad de la pancreatitis. ${ }^{11}$

Se ha definido la pancreatitis lúpica como la presencia de síntomas clínicos típicos (incluyendo dolor abdominal, náuseas y vómitos), con confirmación mediante un aumento mayor a tres veces de la amilasa o la lipasa séricas o evidencia de los estudios de diagnóstico por imagen (TC o 
ecografía) o CPER.7 La Sociedad Pancreática de Japón propuso, en 2002, los siguientes criterios diagnósticos para determinar la pancreatitis autoinmunitaria: 1) estudios de diagnóstico por imagen del páncreas que revelan estrechamiento difuso del conducto pancreático principal con paredes irregulares y agrandamiento difuso del páncreas; 2) datos de laboratorio que indican concentraciones anormalmente elevadas de gamma globulina sérica o IgG o presencia de autoanticuerpos; y 3) examen histopatológico del páncreas que muestra cambios fibróticos con infiltración de células plasmáticas y linfocitos. ${ }^{12}$

Se deben usar corticoesteroides para el tratamiento médico de la pancreatitis aguda asociada a LES; en particular, el tratamiento con corticoesteroides en dosis altas adicionales ha sido satisfactorio en el manejo de la pancreatitis asociada a LES. ${ }^{2,13}$ La paciente descrita en este informe desarrolló pancreatitis aguda asociada a LES concurrente con nefritis lúpica; ambas complicaciones se trataron adecuadamente con corticoesteroides. Los agentes inmunosupresores, como micofenolato mofetilo o ciclofosfamida, pueden usarse en combinación con corticoesteroides. Cabe destacar que las medidas complementarias también fueron sumamente importantes. La paciente mostró valores elevados de proteinuria e hipoalbuminemia, y había recibido alimentación por nutrición parenteral total a través de un PICC. Las medidas de apoyo adecuadas son útiles para facilitar la recuperación funcional y mejorar el desenlace del paciente. ${ }^{2}$ En los casos graves, la plasmaféresis y la infusión intravenosa de gamma globulina podrían ser beneficiosas. ${ }^{13}$ El caso de nuestra paciente sugiere que la metilprednisolona en inyección intravenosa debe administrarse de inmediato si los análisis clínicos y bioquímicos indican que el LES es el causante de la pancreatitis. La ciclofosfamida en inyección intravenosa debe ser la primera opción de tratamiento con agentes inmunosupresores para la pancreatitis asociada a LES. ${ }^{14} \mathrm{El}$ tratamiento temprano y agresivo podría salvar la vida del paciente.

\section{REFERENCIAS}

1. Li Z, Xu D, Wang Z, Wang Y, et al. Gastrointestinal system involvement in systemiclupus erythematosus. Lupus. 2017; 26(11):1127-38.

2. $\mathrm{Tu}$ YL, Yeh KW, Chen LC, Yao TC, et al. Differences in disease features between childhood-onset and adultonset systemic lupus erythematosus patients presenting with acute abdominal pain. Semin Arthritis Rheum. 2011; 40(5):447-54.

3. Limwattana $S$, Dissaneewate P, Kritsaneepaiboon S, Dendumrongsup T, et al. Systemic lupus erythematosusrelated pancreatitis in children. Clin Rheumatol. 2013; 32(6):913-8.

4. Xu D, Yang H, Lai CC, Li P, et al. Clinical analysis of systemic lupus erythematosus with gastrointestinal manifestations. Lupus. 2010; 19(7):866-9.

5. Alves SC, Fasano S, Isenberg DA. Autoimmune gastrointestinal complications in patients with systemic lupus erythematosus: case series and literature review. Lupus. 2016; 25(14):1509-19.

6. Weening JJ, D'Agati VD, Schwartz MM, Seshan S, et al. The classification of glomerulonephritis in systemic lupus erythematosus revisited. J Am Soc Nephrol. 2004; 15(2):241-50.

7. Yang Y, Ye Y, Liang L, Wu T, et al. Systemic-lupuserythematosus-related acute pancreatitis: a cohort from South China. Clin Dev Immunol. 2012; 2012:568564.

8. Makol A, Petri M. Pancreatitis in systemic lupus erythematosus: frequency and associated factors - a review of the Hopkins Lupus Cohort. J Rheumatol. 2010; 37(2):341-5.

9. Wang $\mathrm{CH}, \mathrm{Yao} \mathrm{TC}$, Huang $\mathrm{YL}, \mathrm{Ou}$ LS, et al. Acute pancreatitis in pediatric and adult-onset systemic lupus erythematosus: a comparison and review of the literature. Lupus. 2011; 20(5):443-52.

10. Marques VL, Gormezano NW, Bonfá E, Aikawa NE, et al. Pancreatitis Subtypes Survey in 852 ChildhoodOnset Systemic Lupus Erythematosus Patients. J Pediatr Gastroenterol Nutr. 2016; 62(2):328-34.

11. Chang YJ, Chao HC, Kong MS, Hsia SH, et al. Acute pancreatitis in children. Acta Paediatr. 2011; 100(5):740-4.

12. Members of the Criteria Committee for Autoimmune Pancreatitis of the Japan Pancreas Society. Diagnosis criteria for autoimmune pancreatitis by the Japan Pancreas Society. J Jpn Pan Soc. 2002; 17:585-7.

13. Wang Q, Shen $M$, Leng X, Zeng X, et al. Prevalence, severity, and clinical features of acute and chronic pancreatitis in patients with systemic lupus erythematosus. Rheumatol Int. 2016; 36(10):1413-9.

14. Perrin L, Giurgea I, Baudet-Bonneville V, Deschênes $\mathrm{G}$, et al. Acute pancreatitis in paediatric systemic lupus erythematosus. Acta Paediatr. 2006; 95(1):121-4. 